\title{
Radial density and density evolution of radio galaxies in the Las Campanas redshift survey
}

\author{
J. Machalski and W. Godlowski
}

\author{
Astronomical Observatory, Jagellonian University, ul. Orla 171, 30244 Cracow, Poland \\ Received 23 November 2000 / Accepted 27 February 2001
}

\begin{abstract}
The sample of 1157 radio-identified galaxies from the Las Campanas Redshift Survey (LCRS) (Machalski \& Condon 1999) is used to determine the radial density of both star-forming (starburst) and AGNtype galaxies with the mean redshift of 0.07 and 0.18 , respectively. It is shown that the radial density distribution of these radio galaxies is essentially different from the respective distribution of optical galaxies in the parent LCRS sample. While the optical galaxies reveal a systematic decrease of their spatial density with redshift (which likely is due to some incompleteness of the optical sample increasing with the apparent isophotal magnitude of galaxies), the radio-emitting galaxies exhibit evident positive evolution of their radial density with redshift. Moreover, most of this density evolution is connected to starburst-type galaxies whose evolution can be parametrized by $g(z) \propto(1+z)^{2.5 \pm 0.5}$. No evident density evolution is detected in LCRS AGN galaxies. Correcting the $1.4 \mathrm{GHz}$ luminosity function (RLF) (Machalski \& Godlowski 2000) for the above density evolution in starbursts and assuming its lack in AGN, a pure luminosity evolution $f(z) \propto(1+z)^{2.4 \pm 0.9}$ is found for AGN galaxies after the comparison of the corrected RLF with the local luminosity function based on nearby UGC galaxies (cf. Condon 1989). Oppositely, no significant luminosity evolution in starbursts is found.
\end{abstract}

Key words. galaxies: active - galaxies: evolution - radio continuum: galaxies

\section{Introduction}

The Las Campanas Redshift Survey of galaxies (Shectman et al. 1996, hereafter LCRS), containing over 90000 galaxies to a limiting isophotal magnitude of $18.2-18.5$, of which over 26000 have spectroscopic redshift measured, is very useful for a large statistical study of properties of faint radio emission in the galaxy population lying farther than about $180 \mathrm{Mpc}$ from our Galaxy and the local space around it. Machalski \& Condon (1999; hereafter Paper I) used the 1.4-GHz NRAO-VLA Sky Survey (Condon et al. 1998, hereafter NVSS) to detect radio emission from LCRS galaxies in the sky area of 0.1438 sr and within a space volume of about $10^{8} \mathrm{Mpc}^{3}$. Taking into account a strongly increasing incompleteness of the LCRS sample with increasing isophotal magnitude of galaxies (which could be clearly seen in the number-counts of these galaxies), the radio detection was limited to galaxies with $m_{\text {iso }} \leq 18.0 \mathrm{mag}$. The optical and radio data, supported by the IRAS far-infrared (FIR) data, were then used to classify 1157 detected galaxies by a dominant radio-energy source: starbursts or AGN.

The above sample of 1157 radio galaxies has been used by Machalski \& Godlowski (2000; hereafter Paper II) to

Send offprint requests to: J. Machalski,

e-mail: machalsk@oa.uj .edu.pl derive the $1.4 \mathrm{GHz}$ (radio) luminosity function (hereafter $\mathrm{RLF}$ ) of both star-forming galaxies with the mean redshift $\langle z\rangle \approx 0.07$, and AGN-type ones with $\langle z\rangle \approx 0.18$, as well as for a direct measure of their evolution inferred from the evolutionary models of the entire population of radio sources (e.g. Condon 1984; Dunlop \& Peacock 1990). In a result, the evolution of RLF, in the "translation" form $\rho_{m}(L, z)=g(z)[L / f(z), z \approx 0]$ given by Condon (1984), Saunders et al. (1990) and others, has appeared uncertain at redshifts characteristic for LCRS galaxies. This is caused by a too large uncertainty of the local luminosity functions determined from bright UGC galaxies by Condon (1989) and Cotton \& Condon (1998) which is of the same order as an amount of density $[g(z)]$ and/or luminosity $[f(z)]$ evolution expected from the evolutionary models. However, a direct comparison of the spectral power density and radial density of UGC and LCRS starburst and AGN galaxies with 1.4-GHz luminosities exceeding $10^{22.8} \mathrm{~W} \mathrm{~Hz}^{-1}$ and $10^{24.0} \mathrm{~W} \mathrm{~Hz}^{-1}$, respectively (i.e. galaxies from the brighter part of their RLFs) agreed with a luminosity evolution $f(z) \propto(1+z)^{3 \pm 1}$. An intriguing fluctuation of the spectral power was noticed if AGN-type galaxies more luminous than $10^{24.0} \mathrm{~W} \mathrm{~Hz}^{-1}$ were divided into three redshift ranges. Those limited radio data was not sufficient to specify whether that effect 
reflected some real clustering of radio galaxies, or was only a statistical fluctuation of their spatial density.

In this paper we analyse the radial density of starbursttype and AGN-type radio galaxies in the LCRS sample and compare them with the respective characteristics of optical galaxies in the parent sample. The principal aim of this investigation is to look whether the spatial density of radio-emitting galaxies correlates or not with the respective density of parent optical galaxies, and whether some density evolution is present in the LCRS sample itself putting aside its comparison to the density of bright UGC galaxies. Moreover, the data of Paper I allow to search for such density evolution separately for star-forming and AGN radio galaxies.

As it was shown in Paper I, in over 60 of 100 cases radio emission has been detected from galaxies not fulfilling the photometric selection criteria to be observed spectroscopically (cf. Lin et al. 1996). Thus, we cannot confine our analysis only to galaxies with spectroscopic redshift available, especially if a fraction of galaxies with known redshift strongly decreases with an increase of their apparent isophotal magnitude (as in the case of LCRS). On the other hand, presumed effects of varying radial density or clustering of galaxies can be present at redshifts corresponding to the faintest magnitudes in the LCRS sample. Therefore, we have to ascribe a photometric redshift estimate to the remaining optical galaxies, as it was done for radio-detected galaxies in Papers I and II. This procedure is summarized in Sect. 2. The ascribed redshifts are then used to (1) derive the "parent" radial density function of optical galaxies taking into account all of them, i.e. those with spectroscopic redshift and those with its photometric estimate, and (2) reproduce their optical luminosity function. Comparing this luminosity function with the original one published by Lin et al., we can test a statistical correctness of our photometric redshifts. The method of analysis is described in Sect. 3. First, this method is applied to the entire optical catalog of LCRS galaxies with the aim to derive their radial density function to which the respective functions for different types of radio galaxies can be compared. The resultant radial density and luminosity functions for the optical galaxies are given in Sect. 4.1. In Sect. 4.2, the same method is applied to the radioidentified LCRS starburst and AGN galaxies. The radial densities of optical and radio LCRS galaxies are compared in Sect. 5. The evident density evolution of starbursts with redshift is shown, and its consequences for the "translation" evolution of the RLF are discussed.

\section{Redshift estimates}

The procedure used to evaluate a photometric redshift for radio-identified LCRS galaxies was described in Paper II. In order to estimate redshift for the optical galaxies which were not observed spectroscopically, we investigated the distribution of the absolute isophotal magnitude $M_{\text {iso }}$ of galaxies from the original redshift survey of Shectman et al. The equation used to calculate $M_{\text {iso }}\left(\right.$ and $\left.M_{\mathrm{R}}\right)$ is

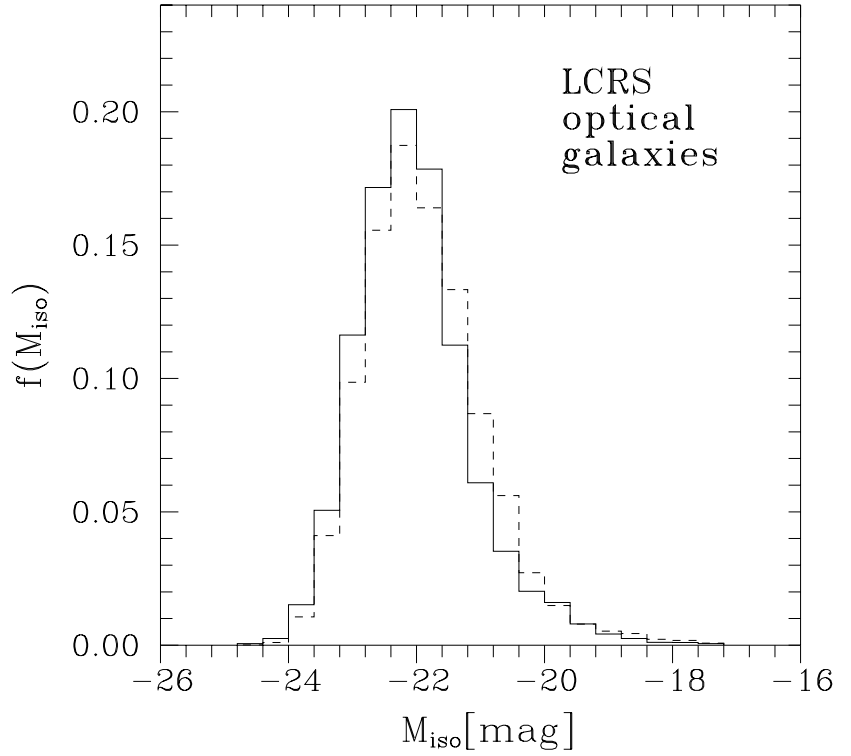

Fig. 1. Absolute optical magnitude of 24089 LCRS galaxies with $14.5 \mathrm{mag} \leq m_{\text {iso }}<16.5 \mathrm{mag}$ (solid line) and with $16.5 \mathrm{mag} \leq m_{\text {iso }}<17.7 \mathrm{mag}$ (dashed line). The mean values and standard deviations in these distributions are $-22.11 \mathrm{mag}$ and $0.94 \mathrm{mag}$, and $-21.89 \mathrm{mag}$ and $0.95 \mathrm{mag}$, respectively

given in Paper I. In that equation the K-correction is calculated with a constant optical spectral index, and an eventual correction for the foreground extinction is neglected because of the high galactic latitudes of the LCRS strips. The distribution of $M_{\text {iso }}$ for 5255 galaxies with $14.5 \mathrm{mag} \leq m_{\text {iso }}<16.5 \mathrm{mag}$ and 18834 with $16.5 \mathrm{mag} \leq m_{\text {iso }}<17.7 \mathrm{mag}$ are shown in Fig. 1. The mean values and standard deviations of both distributions are given in the caption of Fig. 1. Although the means of these two distributions differ only by $0.22 \mathrm{mag}$, the Kolmogorov-Smirnov test showed that this difference is very significant statistically. However, this is a formal difference caused by the simple calculation of the absolute magnitude of a galaxy with a constant optical spectral index equivalent to a constant $B-R$ colour. As a matter of fact, that spectral index evolves with redshift and its evolution can be different for different morphological types of galaxies. In general, the above index steepens with redshift if $B-R>1 \mathrm{mag}$ and due to intrinsic evolution of the stellar content in a galaxy seen at different epochs.

The majority of LCRS galaxies have a redshift in the range $0.05-0.20$. If the evolution of $B-R$ colour within this redshift range for early-type galaxies (E, S0) (e.g. Bruzual 1983) is taken into account, $M_{\text {iso }}$ of galaxies with fainter apparent magnitudes will be enlarged by $0.20 \mathrm{mag}$, which will compensate the apparent difference of 0.22 mag. Obviously, the LCRS sample must contain also a large fraction of spiral galaxies whose spectra are much bluer in comparison to those in ellipticals, and the difference of $0.22 \mathrm{mag}$ will not be fully compensated by the spectral evolution. In that view, LCRS galaxies with fainter $m_{\text {iso }}$ seem to be little less luminous. 
Therefore in order to presume a distribution of $M_{\text {iso }}$ for the galaxies with $17.7 \mathrm{mag}<m_{\text {iso }} \lesssim 18.2 \mathrm{mag}$, we kept the formal increase of $M_{\text {iso }}$ distribution with increasing $m_{\text {iso }}$ (which resulted from the calculation of $M_{\text {iso }}$ with a constant $B-R$ colour) and found that the mean of this distribution should be shifted by another $0.1 \mathrm{mag}$ to the value of about $-21.8 \mathrm{mag}$. Consequently, for each galaxy without spectroscopic redshift and brighter than $17.7 \mathrm{mag}$, an absolute magnitude was drawn by chance from the $M_{\text {iso }}$ distributions in Fig. 1 whereby, for those fainter than $17.7 \mathrm{mag},+0.10 \mathrm{mag}$ was added to $M_{\text {iso }}\left[16.5 \leq m_{\text {iso }}<\right.$ 17.7] and used to calculate the relevant redshift. A statistical error of the radial density solutions due to uncertain redshift is discussed in Sect. 3.

\section{The method}

\subsection{Radial density function}

In order to determine the radial density functions of LCRS galaxies and radio galaxies, we use the "stepwise maximum likelihood" (hereafter SWML) method of Efstathiou et al. (1988) with its modification needed for the analysis of a sample of objects limited both by the radio and optical flux densities, as it was shown in Paper II.

As in Saunders et al., we parametrize $g(r)$ as an arbitrary $N_{z}$ steps in redshift:

$g(z)=g_{k}, \quad z_{k}-0.01 \leq z<z_{k}+0.01, \quad k=1, \ldots, N_{z}$

and maximize the likelihood:

$\ln \mathcal{L}=$

$\sum_{i=1}^{N}\left\{\ln g\left(z_{i}\right)-\ln \left[\sum_{k=1}^{N_{z}} g\left(z_{k}\right) f_{i k} V_{\max , i}\right]\right\}+$ const

$\approx \sum_{k=1}^{N_{z}} n_{k} \ln g_{k}-\sum_{i=1}^{N} \ln \left[\sum_{k=1}^{N_{z}} g\left(z_{k}\right) f_{i k} V_{\max , i}\right]+$ const,

where $N$ is a number of galaxies in the sample, $V_{\max , i}$ is the maximum volume determined by the maximum redshift at which the $i$ th galaxy would have apparent fluxes above both the optical and radio detection limits, and $f_{i k}\left(0 \leq f_{i k} \leq 1\right)$ is the fraction of the step volume contained in the $V_{\max , i}$.

Equating the first derivative of the above equation to zero, the maximum likelihood solution $\left\{g_{k}\right\}$, for each $k$ is

$g_{k}=n_{k}\left\{\sum_{i=1}^{N}\left[f_{i k} V_{k}\left(\sum_{j=1}^{N_{z}} g_{j} f_{i j} V_{\max , i}\right)^{-1}\right]\right\}^{-1}$.

After the differentiation $V_{k}$ is the volume of the $k$ th redshift bin, and $f_{i k}$ in Eq. (3) is the fraction of the $V_{k}$ available to the $i$ th galaxy. Setting the average density to unity, we arbitrary normalize the radial density distributions. The radial density function derived as above describes a density fluctuations of galaxies regardless on their luminosities. In order to stress its relativity for different type galaxies, hereafter we write the normalized radial density function by $g_{n}(z)$.

\subsection{Rendering the luminosity function}

Given the radial density fluctuations, the luminosity function of galaxies can be reconstructed with the simple binned $\sum\left(1 / V_{\max }\right)$ method which (in this case) accounts for these fluctuations. The application of this method to the optical LCRS sample allows to check a correctness of the statistical redshift estimates. Following the Saunders et al. analysis, we sum reciprocals of the "effective" volume $\sum_{j=1}^{N_{z}} g_{j} f_{i j} V_{\max }$ over all galaxies with absolute magnitude fulfilling $M_{k}-\Delta M / 2 \leq M<M_{k}+\Delta M / 2$. That is

$\rho_{k} \equiv \rho\left(M_{k}\right) \Delta M=\sum_{i=1}^{N}\left\{w_{i k}\left(\sum_{j=1}^{N_{z}} g_{j} f_{i j} V_{\max , i}\right)^{-1}\right\}$

where $w_{i k}=1$ if absolute magnitude of the $i$ th galaxy is within the $k$ th absolute magnitude bin, otherwise $w_{i k}=0$.

\subsection{Errors of the solutions}

The estimates of $g_{k}$ and $\rho_{k}$, derived as above, are biased by two statistical errors. The first one is the error of SWML solution which is mainly dependent on the number of galaxies in a bin. This error is strongly asymmetric when the numbers are small, and is calculated like that in Efstathiou et al. and Saunders et al. The second one arises from the redshift error in the calculation of space volume.

Like in Paper II, we have evaluated the second error, performing a large number of Monte Carlo simulations in which, for each galaxy without spectroscopic redshift, a $M_{\text {iso }}$ was drawn by chance from the distributions shown in Fig. 1. The standard deviation of $g_{k}$ and $\rho_{k}$ values in the $k$ th bin, resulted from the above simulations, is taken as the second error. Thus, the total statistical error is calculated combining both errors quadrically.

\section{Radial density of LCRS galaxies}

\subsection{Optical galaxies}

The observed numbers in the redshift bins of 0.02 and the estimates of the normalized $g_{n}(z)$ function for 78298 optical galaxies with $12.25 \mathrm{mag}<m_{\text {iso }}<18.15 \mathrm{mag}$ selected from the LCRS photometric catalog are tabulated in Cols. 2 and 3 of Table 1, respectively, and plotted in Fig. 2. Vertical bars indicate the statistical rms uncertainty (variance) in each of the bin. The $g_{n}(z)$ estimates computed for the galaxies with spectroscopic redshift only (marked by the dots in Fig. 2) emphasize the known incompleteness of the LCRS sample due to the original selection effects dominated by the central surface brightness selection (cf. Lin et al.). The derived radial densities systematically decrease with increasing redshift, at least from $z \approx 0.05$ to $z \approx 0.30$. This decrease seems to continue until $z \approx 0.40$ as the most luminous LCRS galaxies, e.g. with $M_{\text {iso }} \approx-24.3 \mathrm{mag}$, can have $m_{\text {iso }} \leq 18.15 \mathrm{mag}$ at that redshift. 
Table 1. Radial density functions of LCRS optical and radio galaxies arbitrarily normalized

\begin{tabular}{|c|c|c|c|c|c|c|c|c|}
\hline & & OPTICAL & & RADIO & & STARBURSTS & & $\mathrm{AGN}$ \\
\hline$z$ & $N$ & $g_{n}(z)$ & $N$ & $g_{n}(z)$ & $N$ & $g_{n}(z)$ & $N$ & $g_{n}(z)$ \\
\hline 0.01 & 662 & $1.27 \pm 0.07$ & 31 & $0.60_{-0.15}^{+0.20}$ & 30 & $0.14 \pm 0.04$ & 1 & $1.25_{-0.79}^{+2.16}$ \\
\hline 0.03 & 3164 & $1.28 \pm 0.08$ & 103 & $0.77_{-0.13}^{+0.15}$ & 94 & $0.18 \pm 0.03$ & 9 & $1.69_{-0.49}^{+0.69}$ \\
\hline 0.05 & 6653 & $1.37 \pm 0.12$ & 128 & $1.05_{-0.13}^{+0.14}$ & 111 & $0.27 \pm 0.04$ & 17 & $1.28_{-0.29}^{+0.37}$ \\
\hline 0.07 & 9800 & $1.35 \pm 0.13$ & 120 & $1.16_{-0.13}^{+0.14}$ & 85 & $0.31_{-0.04}^{+0.05}$ & 35 & $1.47_{-0.24}^{+0.29}$ \\
\hline 0.09 & 10939 & $1.22 \pm 0.10$ & 103 & $1.13_{-0.14}^{+0.15}$ & 55 & $0.34_{-0.05}^{+0.06}$ & 48 & $1.33_{-0.19}^{+0.25}$ \\
\hline 0.11 & 10972 & $1.15 \pm 0.08$ & 96 & $1.09_{-0.13}^{+0.14}$ & 37 & $0.37_{-0.07}^{+0.08}$ & 59 & $1.24_{-0.16}^{+0.19}$ \\
\hline 0.13 & 10106 & $1.10 \pm 0.08$ & 99 & $1.17_{-0.14}^{+0.15}$ & 31 & $0.58_{-0.11}^{+0.14}$ & 68 & $1.20_{-0.15}^{+0.17}$ \\
\hline 0.15 & 8032 & $1.02 \pm 0.06$ & 93 & $1.12_{-0.14}^{+0.15}$ & 30 & $1.08_{-0.23}^{+0.28}$ & 63 & $0.99_{-0.13}^{+0.15}$ \\
\hline 0.17 & 6166 & $0.98 \pm 0.03$ & 65 & $0.79_{-0.13}^{+0.14}$ & 11 & $0.75_{-0.22}^{+0.30}$ & 54 & $0.77_{-0.10}^{+0.12}$ \\
\hline 0.19 & 4394 & $0.94 \pm 0.02$ & 79 & $0.98_{-0.13}^{+0.14}$ & 8 & $1.19_{-0.40}^{+0.59}$ & 71 & $0.97_{-0.12}^{+0.13}$ \\
\hline 0.21 & 3063 & $0.95 \pm 0.03$ & 66 & $0.88_{-0.14}^{+0.15}$ & 7 & $2.23_{-0.83}^{+1.32}$ & 59 & $0.84_{-0.11}^{+0.13}$ \\
\hline 0.23 & 1892 & $0.89 \pm 0.03$ & 64 & $0.96_{-0.15}^{+0.16}$ & 2 & $1.38_{-0.75}^{+1.61}$ & 62 & $0.97_{-0.13}^{+0.11}$ \\
\hline 0.25 & 1122 & $0.86 \pm 0.04$ & 71 & $1.19_{-0.17}^{+0.18}$ & 1 & $1.54_{-1.04}^{+3.20}$ & 70 & $1.26_{-0.16}^{+0.18}$ \\
\hline 0.27 & 649 & $0.85 \pm 0.06$ & 39 & $1.11_{-0.20}^{+0.22}$ & & & 39 & $1.06_{-0.17}^{+0.20}$ \\
\hline 0.29 & 336 & $0.81 \pm 0.10$ & & & & & & \\
\hline 0.31 & 196 & $0.92_{-0.19}^{+0.20}$ & & & & & & \\
\hline 0.33 & 91 & $0.83_{-0.20}^{+0.22}$ & & & & & & \\
\hline 0.35 & 36 & $0.73_{-0.23}^{+0.25}$ & & & & & & \\
\hline 0.37 & 19 & $0.87_{-0.25}^{+0.27}$ & & & & & & \\
\hline 0.39 & 6 & $0.61_{-0.28}^{+0.33}$ & & & & & & \\
\hline Total & 78298 & & 1157 & & 502 & & 655 & \\
\hline
\end{tabular}

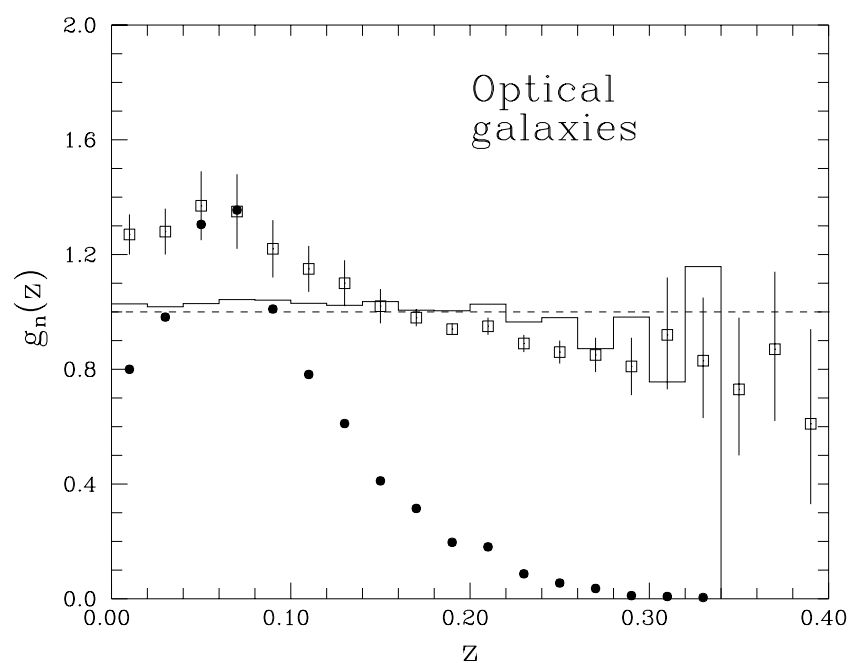

Fig. 2. Arbitrarily normalized radial density of 78298 optical galaxies with $12.25 \mathrm{mag}<m_{\text {iso }}<18.15 \mathrm{mag}$. The continuous line indicates the $g_{n}(z)$ function if these galaxies would be randomly distributed in the space volume (cf. the text). The dots indicate radial density if only galaxies with spectroscopic redshift were taken into account, i.e. 25419 of 78298 galaxies

In order to prove that this decrease is not produced by the method, the same 78298 galaxies with $M_{\text {iso }}$ previously drawn by chance (cf. Sect. 2) were randomly distributed in the space volume limited by the radius equivalent to $z=0.34$. The galaxies which then fell out of a limiting $m_{\text {iso }}$ magnitude (arbitrarily chosen as 18.0 here) were discarded. The $g(z)$ histogram in the redshift bins of 0.02 for the remaining galaxies is shown in Fig. 2 by the continuous

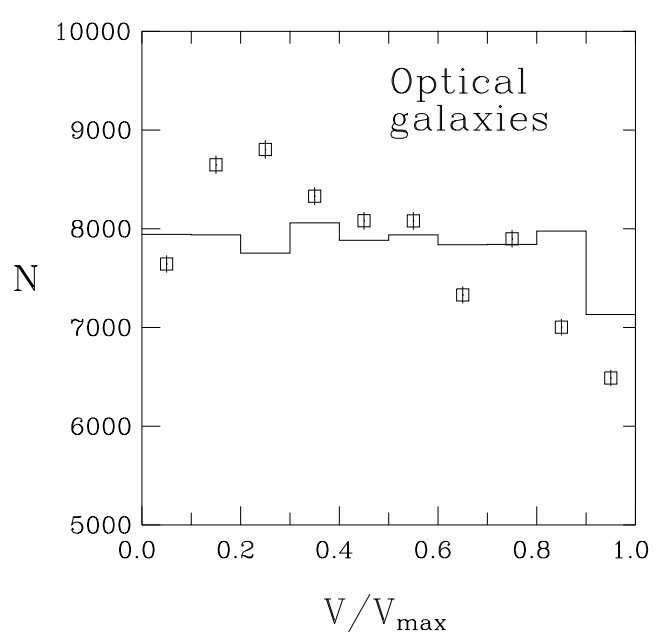

Fig. 3. $V / V_{\max }$ distribution for the same galaxies as in Fig. 2 (open squares). The statistical rms errors marked by the vertical lines are comparable to the size of squares. The continuous line indicates the ratio $V / V_{\max }$ for these galaxies randomly distributed in the space volume

line. The resultant density function is fairly uniform; its larger fluctuations at $z \geq 0.28$ are caused by much smaller statistics there.

The effect of systematic decrease of the radial density with redshift is confirmed by the $V / V_{\max }$ test applied to real LCRS galaxies. $V / V_{\max }$ distribution for the same 78298 galaxies is shown in Fig. 3 and compared with the respective distribution obtained for randomly distributed galaxies. The $V / V_{\max }$ test supports the $g_{n}(z)$ distribution 


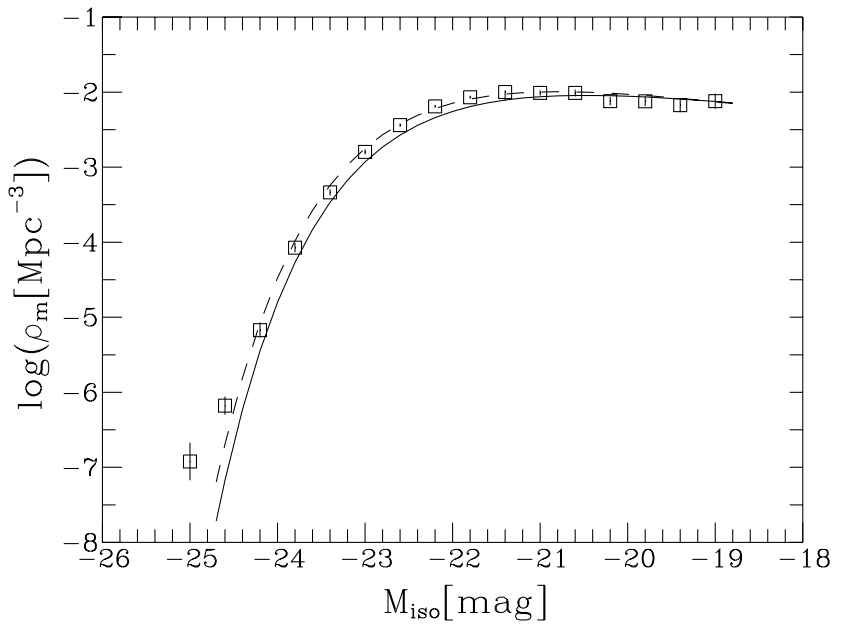

Fig. 4. Density-corrected optical luminosity function for 78298 LCRS galaxies, $67 \%$ of which have the redshift estimated (cf. the text). The squares show the SWML solution plotted with its rms error. The dashed curve shows the bestfitted Schechter function. The solid curve indicates the original luminosity function for the combined NS112 sample of Lin et al.

in Fig. 2 which indicates a maximum of the radial density at $z \approx 0.05-0.07$ and about 40 per cent decrease of it at $z \approx 0.30$.

The radial density of LCRS galaxies derived here contradicts that originally published by Lin et al. (1996), which was determined from spectroscopically observed LCRS galaxies with the use of complicated corrections for the selection effects. If this discrepancy was caused by our (uncorrect) redshift estimates, absolute optical magnitudes of the galaxies without spectroscopic redshift would have a different distribution than those in Fig. 1. To check this possibility, we recalculate the optical luminosity function of all 78298 LCRS galaxies with the prescription given in Sect. 3.2 which takes into account their density fluctuations. The resultant estimates of $\rho\left(M_{\text {iso }}\right)$ in $\Delta M_{\text {iso }}=0.4$ mag bins with their rms errors are plotted in Fig. 4 with the open squares. The solid curve indicates the original luminosity function fitted with the Schechter form by Lin et al. for their combined North + South 112 sample (cf. their Table 2). The dashed curve is the bestfitted Schechter function to our estimates. The relevant parameters are: $M^{*}[\mathrm{mag}]=-20.35 \pm 0.05+5 \log h, \alpha=$ $-0.63 \pm 0.07$, and $\rho^{*}\left[\mathrm{Mpc}^{-3}\right]=0.023 \pm 0.0013$, where $h=H_{\mathrm{o}} /\left(100 \mathrm{~km} \mathrm{~s}^{-1} \mathrm{Mpc}^{-1}\right)$. A point to note is that both luminosity functions in the absolute magnitude range $-24.4 \mathrm{mag}<M_{\text {iso }}<-19.0 \mathrm{mag}$ are consistent within their combined $2 \sigma$ errors.

Thus, we can conclude that a systematic redshift error in our analysis is unlikely, and the decreasing radial density of optical galaxies in the LCRS sample is not caused by erroneous redshift estimates. If not true, this decrease might be due to an incompleteness of the optical sample increasing with the apparent magnitude of galaxies and/or a systematic underestimation of this magnitude in faint galaxies. Our analysis suggests a different weight of these effects in each of the LCRS declination strips. However, an explanation of the above effects is not crucial for the purpose to compare the radial densities of radio-detected galaxies with the respective density of their parent optical population.

\subsection{Radio galaxies}

The observed numbers and the normalized $g_{n}(z)$ estimates found for the radio-detected LCRS galaxies are tabulated in Cols. 4 and 5 of Table 1, respectively, and plotted in Fig. 5. Again, the vertical bars indicate the statistical rms error in each of the bin (as given in Table 1). The similar iterative solutions of the radial density were found separately for the star-forming and AGN galaxies. The observed numbers and the $g_{n}(z)$ estimates for LCRS starbursts and AGN are tabulated in Cols. 6, 7 and 8, 9 of Table 1, respectively. In these two distributions we keep the same normalization as for all radio galaxies. This approach protects an unrealistic increase of $g_{n}(z)$ e.g. for AGN in respect to its value for all galaxies, i.e. AGN+starbursts, however some differences due to numerical iterations in the formal solutions are justified.

\section{Discussion of the results}

\subsection{Comparison of radial densities for optical and radio galaxies}

Comparing the radial density distributions in Figs. 2 and 5 one can easily notice that $g_{n}(z)$ for the radio galaxies differs from that for the optical galaxies. Indeed, the Kolmogorov-Smirnov test indicates that these two distributions are completely independent (they differ at the significance level $\gg 0.9999$. This means that the radioemitting galaxies are not drawn by chance from their optical population. Dividing $g_{n}(z)$ estimates in Col. 5 by these in Col. 3, we can correct the radial density distribution of radio-detected galaxies for the bias introduced by the density of optical galaxies in the parent LCRS sample.

The corrected distribution is shown in Fig. 5 by the staircase line. This histogram clearly has a nonuniform shape and suggests a systematic increase of $g_{n}(z)$ for radiodetected LCRS galaxies. Our analysis shows that this increase is caused mostly by the star-forming galaxies. The $g_{n}(z)$ diagrams for starbursts and AGN are plotted in Figs. 6a and b, respectively.

Surprisingly, these two diagrams are essentially different. While the radial density of starbursts strongly increases with redshift, that for AGN seems to resemble the $g_{n}(z)$ for optical galaxies. Indeed, the correction of their radial density estimates for the optical bias gives almost a uniform distribution indicated by the staircase histogram in Fig. 6b. The above suggest that LCRS starburst-type and AGN-type galaxies have different radial distributions. 


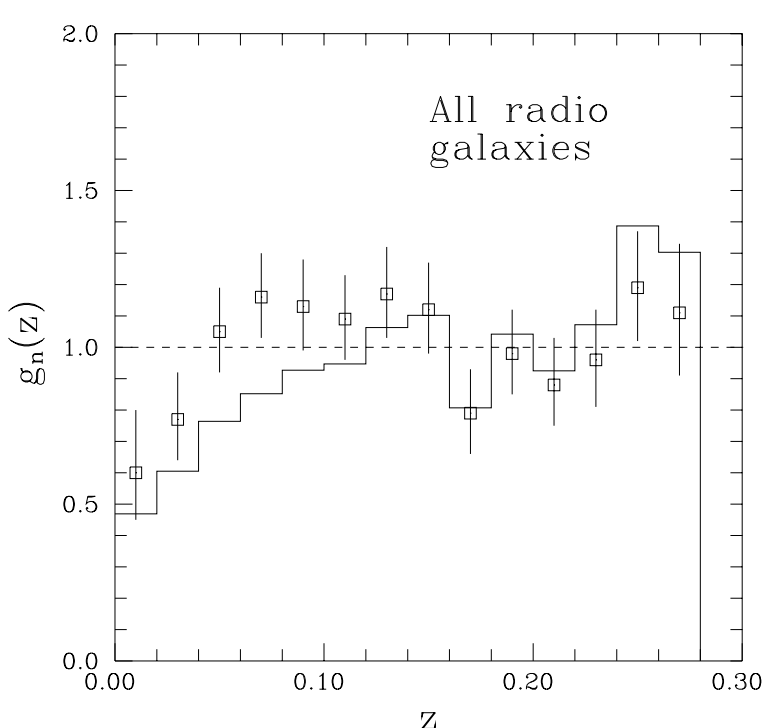

Fig. 5. Arbitrarily normalized radial density of radio-detected galaxies; The statistical rms error in each of the bin is marked by the vertical line. The continuous (staircase) line indicates $g_{n}(z)$ distribution corrected for the bias introduced by the respective distribution for optical galaxies in the parent LCRS sample

\subsection{Density and luminosity evolution}

For the quantitative description of the increase of the radial density with redshift for starbursts (in Fig. 6a), we have assumed a power-law dependence in the form $g_{n}(z) \propto(1+z)^{p}$ and fitted the best power $p$ using the $\chi^{2}$ method. For the reason that the enormous increase of $g_{n}(z)$ at redshifts above $0.15-0.16$ results from the detection of few but very luminous starbursts, we have conservatively fitted $p$ in the redshift range of 0.0 to 0.16 only, i.e. in the range where the radial density is determined by at least 30 galaxies in each of the redshift bins. As the result, we found $p=2.5 \pm 0.5$ suggesting a systematic density evolution of starburst-type galaxies. If the radial density of the LCRS starbursts at $z \gtrsim 0.16$ was really as high as shown in Fig. 6a, their density evolution would be even much stronger. The fit in the redshift range $0.12<z<0.24, p=5.8 \pm 1.1$, is compatible with $p=6.7 \pm 2.3$ obtained by Saunders et al. for IRAS galaxies, but disagrees with the result of Rowan-Robinson et al. (1993) which argued that the density evolution model is inconsistent with faint radio source counts at $1.4 \mathrm{GHz}$. Oppositely, we did not find a noticeable density evolution of LCRS AGN-type galaxies.

Having the radial density distributions of the starburst-type and AGN-type radio galaxies in the LCRS sample, we have recalculated their $1.4 \mathrm{GHz}$ RLF accounting for the variable density additionally corrected for the bias introduced by the respective density distribution of optical galaxies in their parent sample. This was done by inserting the corrected values of $g\left(z_{j}\right)$ into Eq. (4) and solving it for $\rho_{k} \equiv \rho_{m}\left(L_{k}\right)$ (cf. Paper II).

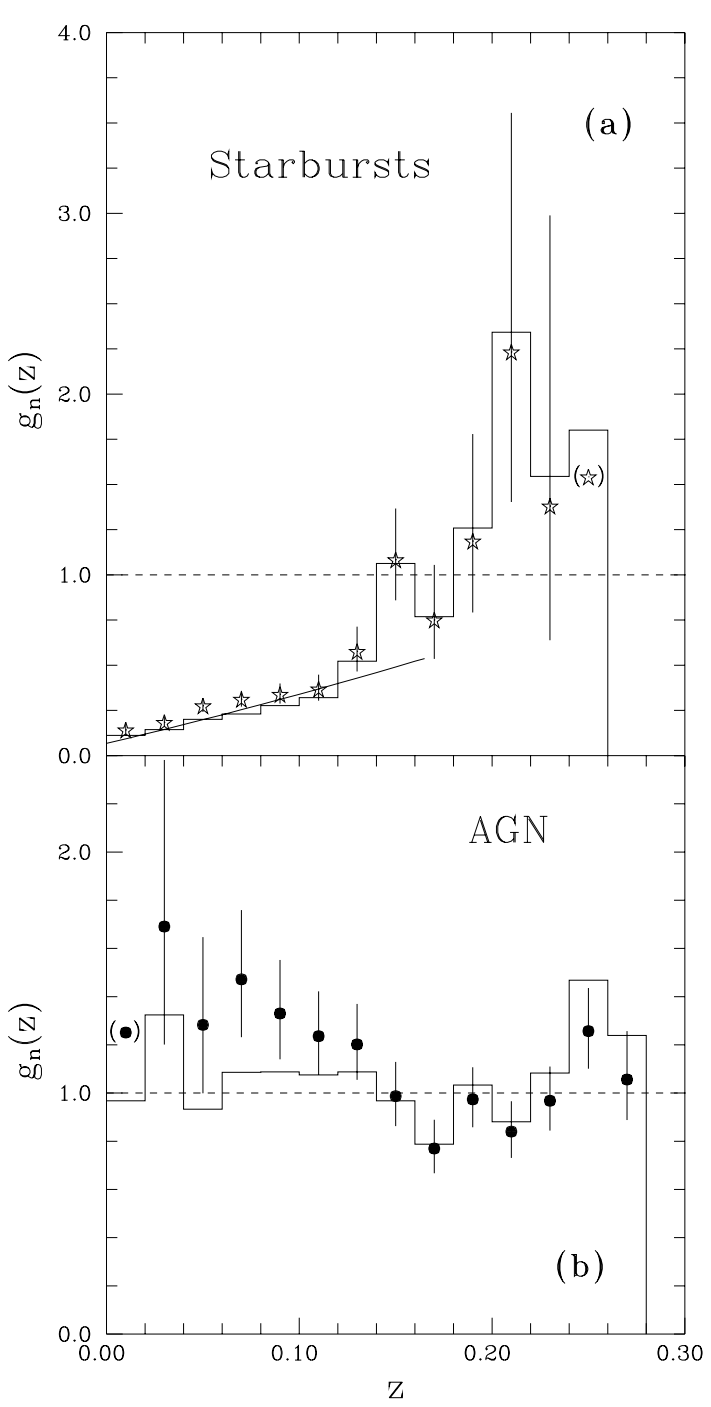

Fig. 6. a) Arbitrarily normalized radial density of Starburst galaxies; b) the same for AGN galaxies. The vertical line in each of the bin and the staircase histograms - as in Fig. 5. Symbols in the parenthesis indicate the density determined by one galaxy only. The solid curve in a) shows the best-fitted power-law dependence in the redshift range of 0.0 to 0.16 : $g_{n}(z) \propto(1+z)^{2.5 \pm 0.5}$

The solved values of $\rho_{k}$, i.e. the comoving space density of galaxies per "magnitude" unit $\log _{m}(L)$, where $m=$ $10^{0.4}$, can be considered as the estimates of the $1.4 \mathrm{GHz}$ RLF corrected for the density evolution at the median redshift of a given sample of LCRS galaxies

$\rho_{m}\left(L, z_{\mathrm{med}}\right) / g\left(z_{\mathrm{med}}\right)=\rho_{m}\left[L / f\left(z_{\mathrm{med}}\right), z \approx 0\right]$.

Given the RLF which includes the density evolution, its eventual luminosity evolution can be found by fitting the local luminosity function (in its logarithmic form) to the $\log \left[\rho_{m}\left(\log L, z_{\text {med }}\right)\right]$ with a horizontal shift in the $\log (L)$ axis

$\log \left[\rho_{m}\left(\log L, z_{\text {med }}\right)\right]=\log \left[\rho_{m}(\log L-\Delta X)\right]$.

The fitted shift $\Delta X$ corresponds to $\log \left[f\left(z_{\text {med }}\right)\right]$. As in Paper II, the local $1.4 \mathrm{GHz}$ luminosity function was 
parametrized using the functional form of Saunders et al. (1990). This form was fitted to the $\rho_{m}(L, z \approx 0)$ estimates for the starburst-type and AGN-type UGC galaxies taken from Condon (1989) and Cotton \& Condon (1998). In the result, $\Delta X=(+0.02 \pm 0.03) \cong \log f(z \approx 0.05)$ and $\Delta X=(+0.17 \pm 0.08) \cong \log f(z \approx 0.18)$ were found for LCRS starbursts and AGN, respectively. For the median redshift of these two types of galaxies, we have adopted their values corrected for varying radial density (given in Sect. 5.2). If the luminosity evolution can be described by $f(z) \propto(1+z)^{n}$, the values of $\Delta X$ correspond to $n=1.0 \pm 0.9$ for starbursts and $n=2.4 \pm 0.9$ for AGN.

Thus we argue that the LCRS starburst-type galaxies exhibit the strong density evolution, at least $g(z) \propto$ $(1+z)^{2.5 \pm 0.5}$, but no certain luminosity evolution. The LCRS AGN galaxies behave oppositely; they show no sign of a density evolution, while their luminosities seem to evolve according to $f(z) \propto(1+z)^{2.4 \pm 0.9}$. The density and luminosity evolution suggested here

(i) do not contradict with the finding shown in Paper II that luminous LCRS starburst and AGN galaxies exhibit some evolution of both the spectral-power density and radial density when compared with the respective local UGC galaxies, though the statistical significance of those results was low due to the poor statistics, especially as concerned more luminous UGC starbursts, and

(ii) seems to confirm some predictions of the evolutionary model of Dunlop \& Peacock (1990) where the lowluminosity sources (a population dominated by normal and star-forming galaxies) and high-luminosity sources (dominated by powerful AGN) can evolve differently. In particular they concluded that "by allowing for a slowly evolving population at low luminosities" all existing observational data could be fitted "with a model whereby luminous sources undergo pure luminosity evolution" if $\Omega_{\mathrm{o}} \cong 1$. It is worth to emphasize that their and our conclusions are compatible though they are drawn from the analyses of essentially different samples: high-redshift radio sources and low-redshift galaxies, respectively.

\subsection{Observed and corrected distributions of redshift}

The effect of varying radial density affects also the observed distributions of redshift for starburst-type and AGN-type LCRS radio galaxies. Dividing the observed number of galaxies in a given redshift bin $N(z)$ (cf. Table 1) by the density distribution derived in Sect. 4 , a corrected redshift distribution is determined

$N_{\text {corr }}(z)=N(z) / g_{n}(z)$.

The comparison between $N(z)$ and $N_{\text {corr }}(z)$ distributions for starburst-type and AGN-type galaxies is provided in Figs. 7a and b, respectively. The corrected distributions, shown there by the dashed curves, are smooth as expected from any model of evolving luminosity function and reveal no trace of the actual density effects. The median redshifts in the corrected distributions are $0.046 \pm 0.003$ and $0.177 \pm 0.004$ for starbursts and AGN, respectively. These

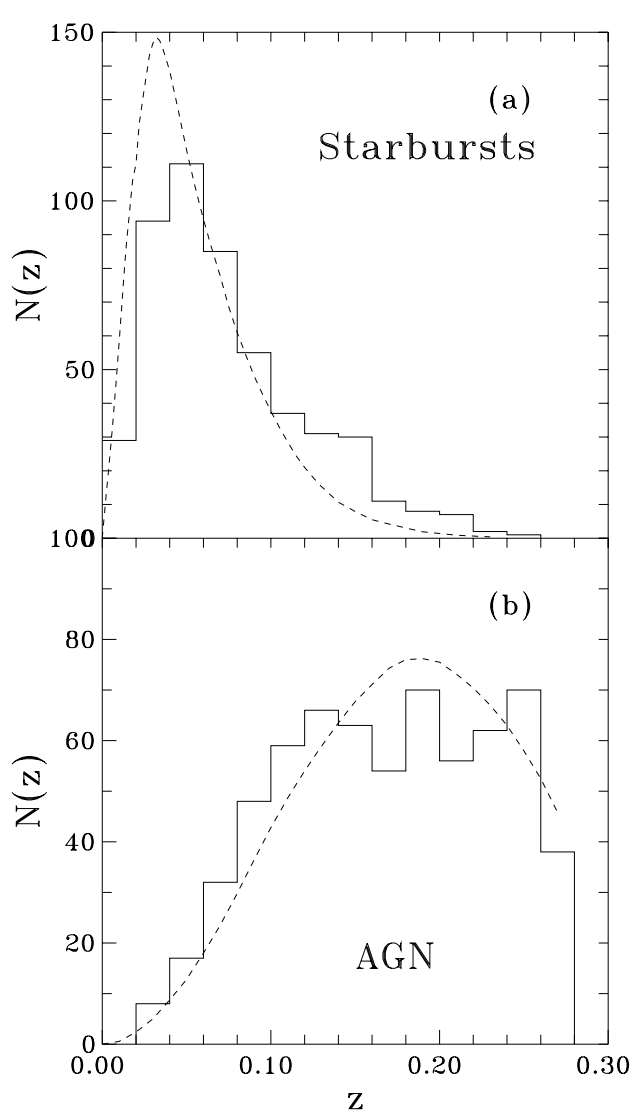

Fig. 7. Histograms of the observed redshift distributions of LCRS galaxies a) for Starbursts, b) for AGN. The dashed curves indicate underlying $N_{\text {corr }}(z)$ distributions after correction for the $g_{n}(z)$ functions given in Table 1

corrected redshift distributions can be very useful for a further observational constraint of the existing models of cosmological evolution of the entire population of radio sources. However this topic is out of scope of the present paper.

\section{Conclusions}

Two main conclusions can be drawn from our analysis:

1) The population of starburst-type galaxies seems to be dominated by the density evolution of a strength at least $g(z) \propto(1+z)^{2.5 \pm 0.5}$, while that of AGN galaxies by the luminosity evolution $f(z) \propto(1+z)^{2.4 \pm 0.9}$. The above conclusion concerning star-forming galaxies fully agrees with that of Mobasher et al. (1999) that "the faint radio sources (in the Phoenix Deep Survey) are not affected by luminosity evolution at $z<0.3$ " [cf. their conclusion (i)].

2) In order to disentangle the effects of density and luminosity evolution at flux densities below $1 \mathrm{mJy}$, deep radio detections of, for example, LCRS galaxies with known redshift would be very fruitful. VLA observations up to $0.2-0.25 \mathrm{mJy}$ beam $^{-1}$ at $1.4 \mathrm{GHz}$, i.e. ten times deeper than the NVSS survey, would allow to detect starbursts more luminous than $10^{22.0} \mathrm{~W} \mathrm{~Hz}^{-1}$ at this frequency up to $z \approx 0.10$. While a maximum of the $1.4 \mathrm{GHz}$ visibility function of starburst-type galaxies is at $L_{1.4} \approx 10^{22.4} \mathrm{~W} \mathrm{~Hz}^{-1}$ 
(cf. Condon 1989; Paper II), suggested observations would guarantee detections of, at least, one half of the entire population of this type galaxies. The results of this paper prove that in a statistically large sample of galaxies, a direct measure of their cosmological evolution is real even at redshifts as low as $0.05-0.07$.

Acknowledgements. This work was supported in part by the State Committee for Scientific Research (KBN) under the contract PB 0266/PO3/99/17.

\section{References}

Bruzual, A. G. 1983, ApJ, 273, 105

Condon, J. J. 1984, ApJ, 287, 461

Condon, J. J. 1989, ApJ, 338, 13

Condon, J. J., Cotton, W. D., Greisen, E. W., et al. 1998, AJ, 115,1693
Cotton, W. D., \& Condon, J. J. 1998, in Observational Cosmology with the NewRadio Surveys, ed. M. N. Bremer, N. Jackson, \& I. Perez-Fournon (Dordrecht: Kluwer), 45 Dunlop, J. S., \& Peacock, J. A. 1990, MNRAS, 247, 19

Efstathiou, G., Ellis, R. S., \& Peterson, B. A. 1988, MNRAS, 232,431

Lin, H., Kirschner, R. P., Shectman, S. A., et al. 1996, ApJ, 464,60

Machalski, J., \& Condon, J. J. 1999, ApJS, 123, 41 (Paper I)

Machalski, J., \& Godlowski, W. 2000, A\&A, 360, 463 (Paper II)

Mobasher, B., Cram, L., Georgakakis, A., \& Hopkins, A. 1999, MNRAS, 308, 45

Rowan-Robinson, M., Benn, C. R., Lawrence, A., McMahon, R. G., \& Broadhurst, T. J. 1993, MNRAS, 263, 123

Saunders, W., Rowan-Robinson, M., Lawrence, A., et al. 1990, MNRAS, 242, 318

Shectman, S. A., Landy, S. D., Oemler, A., et al. 1996, ApJ, 470,172 